\title{
CONCEPTUAL DESIGN UPGRADE ON HYBRID POWERTRAINS RESULTING FROM ELECTRIC IMPROVEMENTS
}

\author{
M. PASSALACQUA ${ }^{1}$, D. LANZAROTTO ${ }^{1}$, M. REPETTO $^{2} \&$ M. MARCHESONI ${ }^{1}$ \\ ${ }^{1}$ Electrical, Electronics and Telecommunication Engineering and Naval Architecture Department (DITEN), \\ University of Genova, Genova, Italy \\ ${ }^{2}$ Department of Mechanical, Energy, Management and Transportation Engineering (DIME), University of Genova, \\ Genova, Italy
}

ABSTRACT

Hybrid vehicles have experienced a great boom in recent years thanks to the increasing spread of 'parallel' architectures, often realized by a planetary gear train (Hybrid Synergy Drive).

At the same time, an enhancement of electrical and electronic components has been experienced; these improvements especially concern reliability and efficiency. Particularly, the possibility of using supercapacitors with increasing storage performances makes possible to manage higher power flows together with a superior efficiency. These innovations may challenge the architecture used nowadays on medium size cars.

The hybrid series architecture, which allows the optimal management of the combustion engine, has been disadvantaged until now by the electric powertrain efficiency.

In the current scenario, this architecture could benefit from the above-mentioned technology, becoming a competitive alternative to the actual powertrain configurations. The aim of this article is the efficiency analysis, in order to evaluate the operational energy efficiency achievable thanks to this configuration. This analysis will be carried out considering all the possible working conditions of the different powertrains.

Keywords: hybrid vehicle, parallel architecture, powertrain efficiency, series architecture, supercapacitor

\section{INTRODUCTION}

The development of hybrid power trains in the automotive industry has been characterized by a large spread of parallel architectures, mostly realized thanks to a planetary gear train [1-3]. A large spread of the series architecture has also taken place as it regards urban bus applications, together with studies supporting and evaluating the benefits deriving from this structure choice $[4,5]$; on the other hand, other studies analyse the effects of different types of storage systems [6]. Series architecture has not seen significant application on the medium size car so far. This type of vehicle for private usage as a matter of fact has to carry out different road missions from one another in terms of average power and maximum power; this fact does not allow the optimization of the internal combustion engine (ICE) generation as it is possible for public transport vehicles where mission power profiles are known a priori.

The wide variability of the power profiles required by vehicles for private usage can be managed, in the series architecture perspective, by storage system appropriately sized. In addition, the energy storing process must be characterized by high efficiency values in order for this solution to be competitive. The aim of this article is to study by simulations the energy flows in series and parallel architectures, especially focusing on the energy cycled by the storage system. Various road missions are taken in consideration, and the results obtained are compared in relation with the particular type of storage system exploited.

Special attention is paid also to the ICE working conditions, the two hybrid architecture allowing in fact different control logic of the power generation. 


\section{POWERTRAIN ARCHITECTURE}

\subsection{Hybrid architecture}

In the technical literature, the hybrid configurations are divided in series and parallel. The architecture equipped with a planetary gear train is conceptually the same as a parallel structure.

\subsubsection{Parallel architecture}

Parallel architecture is shown in Fig. 1, where two different parallel solutions are presented. The two configurations are conceptually identical in that the power provided for the wheels can be the sum of the power coming from the ICE and the power coming from the electric motor. However it is reported in Ref. [7] that the discontinuous variable transmission (Fig. 1 on the right) is more efficient than the other, this is the reason why the comparison carried out in this study between series and parallel architecture does not consider the continuous variable transmission (CVT) configuration.

The heuristic management logic used in this study leads to charge the storage system almost only by regenerative deceleration, this choice is supported by the fact that a storage system charge by ICE is not efficient, in particular the validity of this assumption was tested via simulation.

The energy recovered, thanks to regenerative decelerations, is then exploited for pure electric traction; this traction mode is privileged at low speed where low power demand makes it undoubtedly convenient.

\subsubsection{Series architecture}

Series architecture, shown in Fig. 2, is characterized by the possibility of delivering power to the wheels only through the electric motor. Because of this reason the entire energy generated by the ICE is converted into electrical forma and thus it flows through the electric generator, electric motor and their converters. The instantaneous difference between the power generated by the ICE and that required by the wheels is compensated by the storage system.

All the losses deriving from the electric components can in principle be compensated by a higher efficient power generation of the ICE (this is shown in the results presentation).
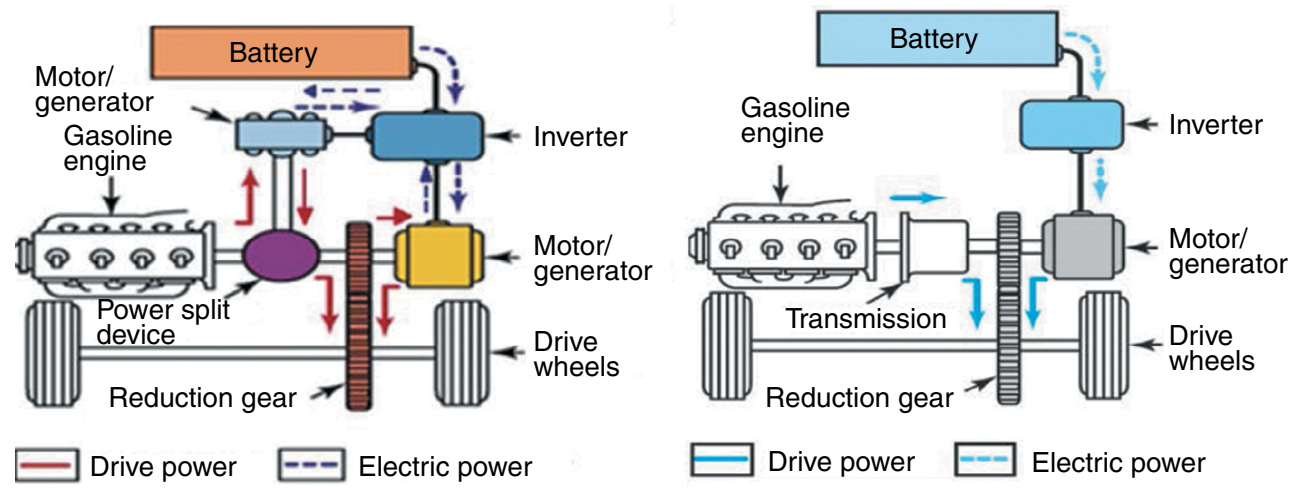

Figure 1: Hybrid parallel architectures. 


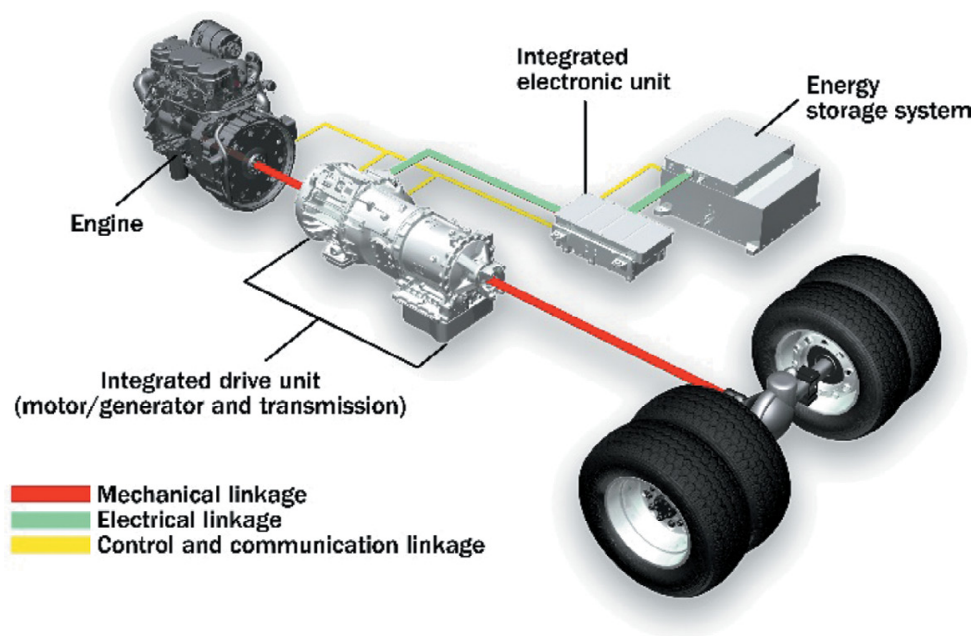

Figure 2: Hybrid series architecture

In particular, this is made possible by the very nature of the series architecture; besides, the possibilities to optimize the ICE working conditions increase with the improvement of the storage system performances.

\subsection{Storage system}

Storage systems have experienced a great development in recent years, both in the transport sector and in stationary applications for electric grids. The most common batteries used in the hybrid automotive industry are the NiMH batteries [1-3], while Li-ion batteries have seen a great technological advance in electric vehicles. Numerous studies pointed out the advantages deriving from a combined use of Li-ion batteries and supercapacitor on medium size cars $[8-10]$ and on urban buses $[6,11]$.

The charge-discharge efficiency of the storage system differs from one technology to another and it depends on the working conditions (mainly current and state of charge, SOC); this is the reason why different values are available in the technical literature even for the same storage type. In Ref. [12], 95\% is the rated efficiency for Li-ion batteries, whereas a simulative study on actual working conditions shows in Ref. [11] an $80 \%$ average efficiency, again for Li-ion batteries. The average efficiency values available in the scientific literature [11-13] for the various technologies are shown in Table 1. These values are to be considered approximate, in that a detailed modelling would be necessary to determine the efficiency in actual working conditions.

Table 1: Storage efficiency.

\begin{tabular}{lccc}
\hline & NiMH & Li-ion & Supercapacitor \\
\hline Efficiency (\%) & $65-80$ & $80-95$ & $97-99$ \\
\hline
\end{tabular}




\section{VEHICLE AND ROAD MISSION FEATURE}

In order to evaluate the performances obtainable with the two architectures described, their models were created in MATLAB/Simulink environment. The vehicle features considered in the simulations are reported in Table 2, while features of different road missions are listed in Table 3; three of these missions are standard drive cycles [14], the data coming from the other three were experimentally acquired in the Genova area. The converter efficiency rates in Table 2 are mean values obtained from dynamic simulations of actual working conditions, and a detailed study of the loss mechanism of these components will be the topic of future studies. Data available in Ref. [15] were used to quantify losses in the electric motor. Finally, the ICE efficiency was acquired from Ref. [16] where a brake-specific fuel consumption map of a spark ignition engine is presented; besides, speed and torque values were normalized and the relative efficiency contour map is plotted in Fig. 3.

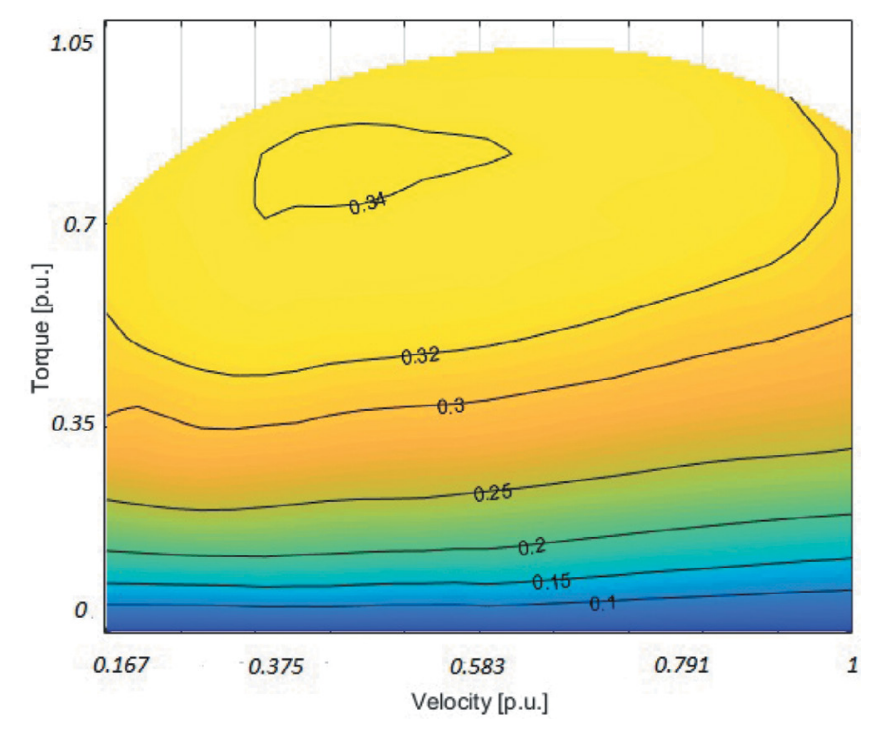

Figure 3: ICE efficiency contour map.

Table 2: Vehicle features.

\begin{tabular}{ll}
\hline Vehicle mass $(\mathrm{kg})$ & 1,450 \\
Rolling coefficient & 0.01 \\
Aerodynamic drag coefficient & 0.25 \\
Vehicle front area $\left(\mathrm{m}^{2}\right)$ & 2.3 \\
Wheel radius $(\mathrm{m})$ & 0.3 \\
Final gear ratio & 8 \\
Differential gear efficiency & 0.97 \\
Transmission efficiency (parallel only) & 0.95 \\
Air density $\left(\mathrm{kg} / \mathrm{m}^{3}\right)$ & 1.22 \\
DC/DC converter efficiency $(\%)$ & 97 \\
Inverter efficiency $(\%)$ & 98 \\
ICE maximum power (parallel/series) $(\mathrm{kW})$ & $72 / 40$ \\
\hline
\end{tabular}


Table 3: Road mission features.

\begin{tabular}{lccccc}
\hline & $\begin{array}{l}\text { Average speed } \\
(\mathrm{km} / \mathrm{h})\end{array}$ & $\begin{array}{l}\text { Maximum speed } \\
(\mathrm{km} / \mathrm{h})\end{array}$ & $\begin{array}{l}\text { Length } \\
(\mathrm{km})\end{array}$ & $\begin{array}{l}\text { Elevation } \\
(\mathrm{m})\end{array}$ & $\begin{array}{l}\text { Maximum road } \\
\text { slope }(\%)\end{array}$ \\
\hline US06 & 78 & 130 & 13 & - & - \\
UDDS & 31 & 90 & 12 & - & - \\
HWFET & 78 & 97 & 16.5 & - & - \\
Urban & 24 & 57 & 11.4 & 20 & Negligible \\
Fast urban & 27 & 68 & 22 & 62 & 6 \\
Extra urban & 45 & 80 & 36 & 300 & 9 \\
\hline
\end{tabular}

4 SIMULATION GOALS

The aim of the simulations is to compare the energy chain efficiency obtainable with the two architectures on different road missions. These efficiency values were computed considering various storage efficiencies, in particular the following values were contemplated: $70 \%, 80 \%$, $90 \%$ and $98 \%$. The goal of this study is not to precisely evaluate the efficiency achievable with the solutions proposed, but it is to quantify the impact the average efficiency of the storage system has on the entire powertrain.

Another aim of these simulations is to establish whether a storage system purely supercapacitor based can be applied to these architectures. As a matter of fact, applications and studies that have been conducted so far only deal with a combined use of batteries and supercapacitors (not used as main storage device). In order to address this matter, two supercapacitor modules were sized differently for the two architectures: a $50 \mathrm{~kg}$ module for the parallel architecture and $85 \mathrm{~kg}$ for the series architecture, thus being respectively characterized by a rated energy of 270 and $470 \mathrm{Wh}$ [17].

Finally, simulations are important to monitor the ICE working conditions. As for the parallel architecture, the ICE control logic implemented refers to those used for vehicles actually on the market $[7,18]$; in the series architecture it was chosen to make the ICE operate only in a small area associated with maximum efficiency. Therefore, on the one hand, the series architecture generates additional losses, but on the other hand it allows the ICE to work at optimal working conditions in terms of torque and speed.

It is thus of primary importance an overall analysis of electric components and ICE efficiency in order to determine the energy virtuosity of this configuration.

\section{SIMULATION RESULTS}

The first important result is given by the fact that the supercapacitor modules never reached the $100 \%$ SOC. This demonstrates how this innovative storage system is suitable for hybrid vehicle applications if properly sized and managed by a correct control logic, despite their low specific energy.

It was then evaluated the chain energy efficiency over the various missions, which is defined as

$$
\eta_{\text {chain }}=\frac{\text { energy necessary to overcome friction }}{\text { primary energy (fuel) }}
$$


Table 4: Chain energy efficiency.

\begin{tabular}{lcccccccc}
\hline$\eta$ storage & \multicolumn{2}{c}{$70 \%$} & \multicolumn{2}{c}{$80 \%$} & \multicolumn{2}{c}{$90 \%$} & \multicolumn{2}{c}{$98 \%$} \\
\hline & Par & Ser & Par & Ser & Par & Ser & Par & Ser \\
US06 & 22.9 & 20.6 & 23.2 & 22.1 & 23.4 & 24.1 & 23.7 & 26.0 \\
UDDS & 16.1 & 15.0 & 16.6 & 17.6 & 16.9 & 20.0 & 17.3 & 22.1 \\
HWFET & 24.5 & 22.1 & 24.6 & 23.9 & 24.7 & 25.6 & 24.8 & 27.5 \\
Urban & 14.7 & 11.1 & 15.6 & 13.5 & 16.3 & 15.5 & 17.2 & 18.3 \\
Fast urban & 16.6 & 13.4 & 17.1 & 15.6 & 17.7 & 17.6 & 18.4 & 20.1 \\
Ex. urban & 19.3 & 14.6 & 19.7 & 17.0 & 20.3 & 19.3 & 20.8 & 22.2 \\
Average & 19.0 & 16.1 & 19.5 & 18.3 & 19.9 & 20.4 & 20.4 & 22.7 \\
\hline
\end{tabular}

The powertrain efficiency for the different architectures, road missions and storage systems is shown in Table 4.

The average efficiency is plotted in Fig. 4. One can immediately notice that series architecture becomes more efficient than the parallel in the high electric component efficiency area (note that the boundaries between the different storage technologies are to give a simple idea of the phenomenon, in fact as previously described their efficiency values change with their working conditions).

To support this consideration, an average Li-ion battery $88 \%$ efficiency value is to consider optimistic [11].

Figure 4 does not take into account economical aspects; series architectures require a much more 'intense' use of the storage system which causes the necessity of replacing periodically the battery. Therefore, it seems that only the realization of this architecture, thanks to supercapacitors (which are characterized by long life in terms of the number of cycles), is able to create a competitive product on the market. This fact explains why this solution has not been commercialized until now for medium size car, where NiMH batteries [1-3] make the parallel structure more convenient.

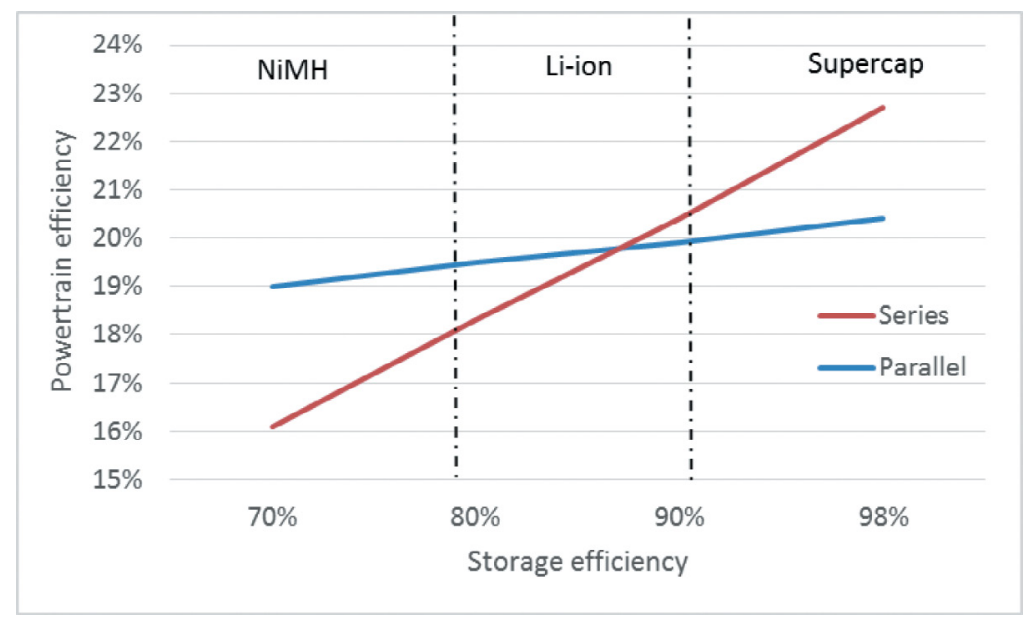

Figure 4: Powertrain average efficiency versus storage efficiency. 
It is clear from the above-mentioned results as to how series architectures offer superior performances compared with the parallel thanks to a supercapacitor base storage system. These advantages are possible although this architecture generates in any case additional electrical losses (losses in the generator, motor, inverters, DC/DC converter and storage system). These losses are as a matter of fact compensated by a higher power generation. As previously mentioned, series architectures allow an optimal management of the ICE working conditions; ICE efficiency and ICE average torque are presented in Table 5, whereas the number of thermodynamic cycles per kilometre is plotted in Fig. 5. The reference ICE is a four-stroke engine with four cylinders, thus the thermodynamic cycles were evaluated multiplying the ICE rounds by 2 .

The simulation results in Table 5 and Fig. 5 explain the reason for series architectures to be more efficient than parallels; the ICE can continuously work at its maximum efficiency rate point (around $80 \mathrm{Nm}$ torque and 2,500 rpm), in that, the energy balance is instantaneously guaranteed by the storage system. On the contrary, in the parallel architecture, the necessity to follow the power profile required by the wheels leads the ICE to work in areas distant from the optimal zone (this happens also for parallel CVT solutions [7]).

As a matter of fact, the ICE works at low loading conditions (lower torque); as a consequence, a higher number of thermodynamic cycles are needed to deliver the same power to the wheels. The number of cycles, being strongly related to mechanical losses, allows one to understand that these losses are much higher in the parallel architecture than in the series.

This fact is particularly evident in urban missions where the rounds per kilometre are three times higher, being equal the energy delivered to the wheels. In addition to what already

Table 5: ICE working condition, average values on the different road missions.

\begin{tabular}{lccc}
\hline ICE efficiency & \multicolumn{3}{c}{ ICE average torque } \\
\hline Parallel (\%) & Series $(\%)$ & Parallel (Nm) & Series (Nm) \\
27.9 & 34 & 46 & 80 \\
\hline
\end{tabular}

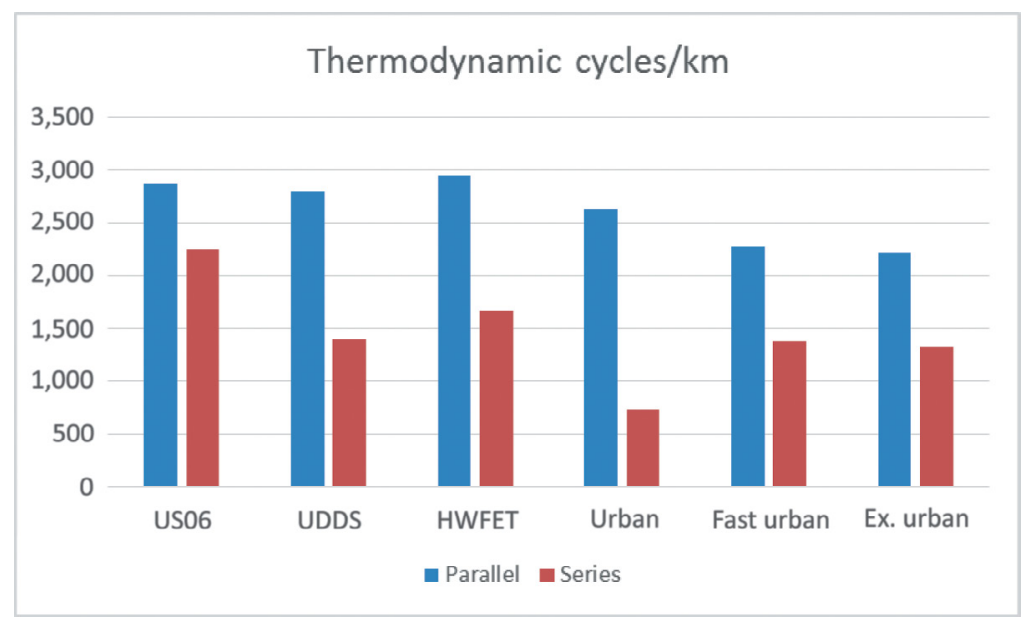

Figure 5: Thermodynamic cycles/km over different road missions. 
mentioned, it is interesting to notice that the engines used in two architectures can be of different power sizes (in this specific study, $72 \mathrm{~kW}$ for the parallel and $40 \mathrm{~kW}$ for the series). Hence, the possibility given by series architectures to downsize the ICE leads once more to lower mechanical losses (mechanical losses not only vary with the number of rounds per minute but also with the engine cylinder capacity).

\section{CONCLUSIONS}

Two MATLAB/Simulink models were created in this study for the energy analysis of parallel and series architectures. Simulations showed that the current state of the art as far as supercapacitors are concerned allows to design a new type of storage system for hybrid vehicles only based on this technology and characterized by masses and dimensions comparable with the batteries today already used.

In particular, this new technology can re-evaluate series architectures for medium size car. Simulations also showed that batteries penalize series architectures, allowing the parallel to be the most efficient solution (hence the large spread of this structure on medium size car).

The models developed here explains why a highly efficient storage system makes the series architecture the most convenient; the optimal management of ICE working conditions allows to generate power more efficiently and with a lower number of thermodynamic cycles.

One step further in the improvement of ICE performances is the introduction of turbocompound systems [5]. This technology can benefit from the high average torque in series architectures, the advantages would then be higher from the introduction of turbocompounds in series systems than in parallels.

This study allows to evaluate what technologies can lead to a significant change in the hybrid architectures for medium size vehicles. However, given the relevant impact of electric components (generator and motor) and of the power electronics on the overall efficiency, an in-depth study of the efficiency of all these components over various working conditions is of primary importance to properly evaluate the fuel economy obtainable in different missions.

Given the results shown earlier, a deeper study of the electric components necessary to realize competitive series architecture will be the content of next studies.

\section{ACKNOWLEDGEMENTS}

The authors would like to wholeheartedly thank Prof. Alessandro Pini Prato for his suggestions, assistance and encouragement throughout this work.

\section{REFERENCES}

[1] Burress, T.A., Campbell, S.L., Coomer, C.L., Ayers, C.W., Wereszczak, A.A., Cunningham, J.P.,et al., Evaluation of the 2010 Toyota Prius Hybrid Synergy Drive System, Power Electronics and Electric Machinery Research Facility, Technical Report ORNL/ TM2010/253, Oak Ridge National Laboratory (ORNL), Oak Ridge, Tennessee, 2011.

[2] Burress, T.A., Coomer, C.L.,Campbell, S.L., Seiber, L.E., Marlino, L.D., Staunton, R.H., et al., Evaluation of the 2007 Toyota Camry Hybrid Synergy Drive System, ORNL/TM2007/190, UT-Battelle, Oak Ridge National Laboratory, Oak Ridge, Tennessee, Revised April 2008.

[3] Burress, T.A., Coomer, C.L., Campbell, S.L., Wereszczak, A.A., Cunningham, J.P. \& Marlino, L.D., Evaluation of the 2008 Lexus LS 600h Hybrid Synergy Drive System, ORNL/TM-2008/185, UT-Battelle, Oak Ridge National Laboratory, Oak Ridge, Tennessee, January 2009. 
[4] Chen, J., Du, J. \& Wu, X., Fuel economy analysis of series hybrid electric bus with idling stop strategy. 2014 9th International Forum on Strategic Technology (IFOST), Cox's Bazar, Bangladesh, pp. 359-362, 2014.

[5] Dellacha, J., Repetto, M., Passalacqua, M., Lanzarotto, D. \& Prato, A.P., Turbocompound technology on hybrid power trains: urban buses take inspiration from F1 power units. Energy Production and Management in the 21st Century II: The Quest for Sustainable Energy, 205, pp. 179-190, 2016. DOI: 10.2495/EQ160171.

[6] Zhao, Y., Yao, J., Zhong, Z.M. \& Sun, Z.C., The research of powertrain for supercapacitor-based series hybrid Bus. 2008 IEEE Vehicle Power and Propulsion Conference, Harbin, China, pp. 1-4, 2008.

[7] Lanzarotto, D., Passalacqua, M. \& Repetto, M., Energy comparison between different parallel hybrid architecture. WIT Energy and Sustainability 2017 (Publication Underway), Seville, 2017 (not yet published).

[8] Camara, M.B. \& Dakyo, B., Energy management for hybrid electric vehicles using load power fluctuation compensation - ultracapacitors and lithium-battery. 2015 Intl Aegean Conference on Electrical Machines \& Power Electronics (ACEMP), 2015 Intl Conference on Optimization of Electrical \& Electronic Equipment (OPTIM) \& 2015 Intl Symposium on Advanced Electromechanical Motion Systems (ELECTROMOTION), pp. 46-51, 2015.

[9] Oukkacha, I., Camara, M.B. \& Dakyo, B., Electric vehicles energy management using lithium-batteries and ultracapacitors. 2017 Twelfth International Conference on Ecological Vehicles and Renewable Energies (EVER), Montecarlo, Monaco, pp. 1-8, 2017.

[10] Mapelli, F.L. \& Tarsitano, D., Energy control for Plug-In HEV with ultracapacitors lithium-ion batteries storage system for FIA Alternative Energy Cup Race. 2010 IEEE Vehicle Power and Propulsion Conference, Lille, France, pp. 1-6, 2010.

[11] Hu, X., Murgovski, N., Johannesson, L.M. \& Egardt, B., Comparison of three electrochemical energy buffers applied to a hybrid bus powertrain with simultaneous optimal sizing and energy management. IEEE Transactions on Intelligent Transportation Systems, 15, pp. 1193-1205, 2014. DOI: 10.1109/TITS.2013.2294675.

[12] Conte, M., Graditi, G., Ippolito, M.G., Sanseverino, E.R., Telaretti, E. \& Zizzo, G., Analisi e definizione di strategie di gestione e controllo di sistemi di accumulo elettrico per applicazioni in reti di distribuzione attive automatizzate, ENEA: Agenzia Nazionale per le Nuove Tecnologie, l'Energia e lo Sviluppo Economico Sostenibile, 2011.

[13] Swal, N. \& Shrivastava, V., An improve efficiency of Li-ion batteries using optimization technique. 2016 3rd International Conference on Computing for Sustainable Global Development (INDIACom), New Delhi, India, pp. 2279-2284, 2016.

[14] EPA, available at https://www.epa.gov/vehicle-and-fuel-emissions-testing/dynamometer-drive-schedules (accessed on 6 October 2017).

[15] Pellegrino, G., Vagati, A., Guglielmi, P. \& Boazzo, B., Performance comparison between surface-mounted and interior PM motor drives for electric vehicle application. IEEE Transactions on Industrial Electronics, 59, pp. 803-811, 2012. DOI: 10.1109/ TIE.2011.2151825.

[16] Konigstein, A., Grebe, U.D., Wu, K.J., Larsson, P.-I., Differenzierte analyse von downsizing-konzepten. MTZ Motortech Z, 69, p. 468, June 2008. DOI: 10.1007/BF03227458.

[17] Maxwell Supercapacitor, available at www.maxwell.com (accessed on 6 October 2017).

[18] Adachi, S. \& Hagihara, H., The Renewed 4-Cylinder Engine Series for Toyota Hybrid System. na, 2012 (from google scholar). 